\title{
Review Article \\ Retinal Imaging of Infants on Spectral Domain Optical Coherence Tomography
}

\author{
Anand Vinekar, ${ }^{1}$ Shwetha Mangalesh, ${ }^{1,2}$ Chaitra Jayadev, ${ }^{1}$ \\ Ramiro S. Maldonado, ${ }^{2}$ Noel Bauer, ${ }^{3}$ and Cynthia A. Toth ${ }^{2}$ \\ ${ }^{1}$ Narayana Nethralaya Postgraduate Institute of Ophthalmology, Bangalore, India \\ ${ }^{2}$ Department of Ophthalmology, Duke University, Durham, NC, USA \\ ${ }^{3}$ Department of Ophthalmology, Maastricht University, Netherlands \\ Correspondence should be addressed to Anand Vinekar; anandvinekar@yahoo.com
}

Received 19 March 2015; Accepted 30 May 2015

Academic Editor: Atsushi Mizota

Copyright (C) 2015 Anand Vinekar et al. This is an open access article distributed under the Creative Commons Attribution License, which permits unrestricted use, distribution, and reproduction in any medium, provided the original work is properly cited.

\begin{abstract}
Spectral domain coherence tomography (SD OCT) has become an important tool in the management of pediatric retinal diseases. It is a noncontact imaging device that provides detailed assessment of the microanatomy and pathology of the infant retina with a short acquisition time allowing office examination without the requirement of anesthesia. Our understanding of the development and maturation of the infant fovea has been enhanced by SD OCT allowing an in vivo assessment that correlates with histopathology. This has helped us understand the critical correlation of foveal development with visual potential in the first year of life and beyond. In this review, we summarize the recent literature on the clinical applications of SD OCT in studying the pathoanatomy of the infant macula, its ability to detect subclinical features, and its correlation with disease and vision. Retinopathy of prematurity and macular edema have been discussed in detail. The review also summarizes the current status of SD OCT in other infant retinal conditions, imaging the optic nerve, the choroid, and the retinal nerve fibre in infants and children, and suggests future areas of research.
\end{abstract}

\section{Introduction}

Optical coherence tomography (OCT) is a routine diagnostic imaging method used worldwide in the evaluation of vitreoretinal diseases in the adult population and has become the indispensable cornerstone of posterior segment disease management over the past few decades. Despite its unquestioned utility in adult clinical practice, it has taken much longer to be used, explored, and understood in pediatric retinal imaging.

In particular, spectral domain OCT (SD OCT) imaging of infants is a relatively new field and has opened up new areas of clinical research in our understanding of the anatomy, as well as evaluating the pathology in these eyes. The aim of this review is to summarize some of the most current and relevant literature on SD OCT imaging of infants and to discuss current and future trends in this field.

Spectral versus Time Domain OCT. Spectral domain optical coherence tomography (SD OCT) measures the interference spectrum of time delay echoes of light using a spectrometer.
This device is based on a high-speed charge coupled device (CCD) camera. Interference spectrum is based on the principle of oscillations and is proportional to the reflected time delay. These scans are produced by a mathematical operation, which extracts the frequency content of this signal. The extracted measure is called the Fourier transformation. The main advantage of SD OCT over the previous technology of time domain OCT or TD OCT is that the former provides better resolution and faster acquisition speeds. This makes it more efficient especially in pediatric imaging, where accurate scans must be obtained in the shortest period of time. Between 25,000 and 100,000 A scans are routinely possible using SD OCT which is $>100$ times faster than the TD devices allowing image capture even in the uncooperative neonate [1].

\section{Historical Aspects}

Historically, the chief limitation of imaging infants with OCT has been the lack of available equipment designed 
to allow quick and easy acquisition of images especially in these unanesthetized, uncooperative, preverbal patients. The limited popularity of SD OCT imaging in infants and young children has been because of the dependence on older equipment that did not allow easy image acquisition in the supine infant. Pediatric patients often needed to be anesthetized or sedated, in an operating room with a team comprised of a pediatric nurse and anesthetist. To allow easier examinations, several modifications have been described, such as, the "flying baby" position which allowed the infant to be horizontally held, albeit under monitoring in the operating room. This allowed images to be acquired upright without the problem of lateral inversion [2].

Previously, the tabletop OCT device which is a vertical imaging system has been converted to a hand-held system for supine imaging $[3,4]$. This has been described using the Spectralis (Heidelberg Engineering, Germany) and is accomplished in a two-step disassembly which frees the camera, allowing the user to align the axis in any plane for capture [4]. Since the infant is supine and needs no anesthesia, this can be performed in the office as well, although monitoring by an anesthetist is advisable. However it must be remembered that since the position of the infant's head is closer to the camera and the technician stands at the head end of the infant, the resulting images are inverted. This is important while interpreting localization of the lesion under study. Using a full-fledged adult device like the Spectralis allows the added advantage of performing fluorescein or indocyanine green angiography simultaneously while acquiring the OCT images [4].

More recently, with the commercial availability of a handheld OCT device, Envisu (Bioptigen, NC, USA), imaging of infants and children has become simpler. Using this device, infants can be imaged conveniently in the office without anesthesia [4-9] or in the operating room [10, 11], as the clinical situation demands. The device allows for the reference arm to be switched between the anterior and posterior segment. The focus is adjusted manually using the noncontact camera and can be performed even through an undilated pupil. The manufacturer-supplied calibration factor allows the conversion of the reference arm read-out to an optical distance measured in millimeters and provides a focus correction with a range of +10 to -12 diopter (D).

\section{Optimizing Image Acquisition}

While considering imaging of pediatric eyes on the SD OCT, we must understand that these eyes are unique in refractive growth and, hence, optics is an important consideration for optimized image capture.

Important differences of the infant eye are as follows:

(1) The axial length increases rapidly in the neonatal period and grows approximately $0.16 \mathrm{~mm}$ per week $[12,13]$. This growth slows with age from approximately $1 \mathrm{~mm}$ /year during the first 2 years to $0.4 \mathrm{~mm} /$ year from 2 to 5 years and to $0.1 \mathrm{~mm} /$ year from 5 to 15 years. After age 15, no significant further growth occurs.
(2) Similarly, the refractive error (RE) varies with age and differs from an adult. Cook et al. reported an RE of $-2.00 \mathrm{D}$ at 32 weeks and -1.23 at 36 weeks, with a shift to hyperopia $(+0.74$ to +2.12$)$ by 40 to 52 weeks

(3) The neonate's cornea is steeper than the adult's cornea, with a mean central corneal power between 48 and $58.5 \mathrm{D}$, decreasing to adult values by three months [13].

(4) Astigmatism is also noted to be greater than in adult eyes and decreases by $50 \%$ in approximately 6 months [14].

(5) While imaging the retina using adult eye settings, the OCT scanning pivot location is displaced anteriorly relative to the pupil, and the peripheral portion of the image is clipped causing loss of peripheral information. This may be overcome by shortening the OCT reference arm delay such that the pivot point is returned to the pupil plane.

Considering the deviation in optics of an infant eye compared to standard adult eye optics, Maldonado et al. recommended age-specific considerations in the SD OCT imaging protocol for young children, which is summarized in Table 1 [8]. This included changing the reference arm position, focus, and scan settings based on age. For example, in a 32-week postmenstrual age (PMA) infant, each millimeter of presumed scan length would actually be $0.629 \mathrm{~mm}$ at the retina ( $62.9 \%$ of the adult eye). Therefore, performing a $10 \mathrm{~mm}$ retinal scan (set for an adult eye) would result in a $6.3 \mathrm{~mm}$ retinal scan in this infant's eye.

\section{Understanding Normal Foveal Development through SD OCT}

SD OCT has offered us an in vivo imaging tool to study the different layers of the infant fovea. This is based on the intrinsic reflectance property of tissues or the interface between adjacent layers. In the retina, the contrast between alternating layers of lower reflective cell nuclei and higher reflective axons, dendrites, and melanosomes allows easy differentiation [15].

Before we understand the development of the fovea in infants, we must be familiar with some definitions used to describe the layers and zones (Figure 1):

(1) Central foveal thickness (CFT, yellow line) which is the thickness of the entire retina from the inner aspect of the inner limiting membrane (ILM) to the inner aspect of the retinal pigment epithelium (RPE) at the foveal center.

(2) Inner retinal layers (IRLs, blue line) which includes all retinal tissue from the inner aspect of the ILM to the outer border of the inner nuclear layer (INL).

(3) The outer retinal layers (ORLs, white line) which extends from the inner aspect of the outer plexiform layer (OPL) to the inner border of the RPE. 
TABLE 1: Reference table for refractive error, axial length, change in reference arm position, and the relative length to an adult scan.

\begin{tabular}{lccccc}
\hline Group age & $\begin{array}{c}\text { Refractive } \\
\text { error (D) }\end{array}$ & Axial length $(\mathrm{mm})$ & $\begin{array}{c}\text { Increase in } \\
\text { reference arm } \Delta \\
\text { Gen 3 engine } \\
\text { (Readout units) }\end{array}$ & $\begin{array}{c}\text { Increase in reference } \\
\text { arm } \Delta / 2 \text { Gen 3+ engine } \\
\text { (Readout units) }\end{array}$ & $\begin{array}{c}\text { Relative scan length to } \\
\text { adult scan length }(\%)\end{array}$ \\
\hline $30-35$ wks & -1 & 15.1 & 97 & -48 & 63 \\
$35-39$ wks & 0.3 & 16.1 & 86 & -43 & 67 \\
$39-41$ wks & 0.4 & 16.8 & 79 & -39 & 70 \\
$0-1$ mos & 0.9 & 17.4 & 72 & -36 & 78 \\
$1-2$ mos & 0.3 & 18.6 & 59 & -29 & 79 \\
$2-6$ mos & 0.5 & 18.9 & 56 & -26 & 80 \\
$6-12$ mos & 0.6 & 19.2 & 52 & -21 & 84 \\
$12-18$ mos & 0.7 & 20.1 & 43 & -15 & 89 \\
18 mos-2 yrs & 0.9 & 21.3 & 30 & & 78 \\
\hline
\end{tabular}

${ }^{*}$ With respect to Gen 3 Engine (Older Bioptigen system), a higher number is equal to a shorter reference arm length on each system used.

${ }^{¥}$ The per turn change in reference path length is two times that of the Gen 3 reference arm. The direction is reversed so that larger numbers refer to larger distances.

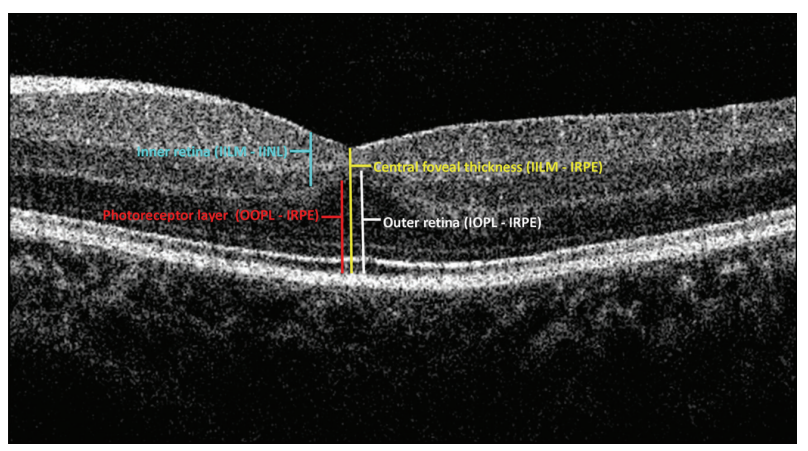

FIGURE 1: The zones and the layers marked on the SD OCT image of a neonate.

(4) The photoreceptor layer (PRL, red line) which extends from the outer aspect of OPL to the inner border of RPE.

Compared to adult foveae, preterm infant foveae differ on SD OCT by demonstrating

(1) a visibly shallower foveal depression;

(2) presence of persisting inner retinal layers including the inner plexiform and the inner nuclear layers (the so called "inner retinal immaturity");

(3) thinner retinal layers overall;

(4) attenuation of the PRL with absence of photoreceptor sublayers.

The retinal layers imaged on SD OCT are compared between a 38-week-old premature infant and a 38-year-old adult in Figure 2.

4.1. Inner Retinal Development. The persistence of the IRLs in the foveal center is characterized by the presence of the ganglion cell layer (GCL), inner plexiform layer (IPL), and the INL as distinct measurable layers at the foveal center. These condense into a single thin hyperreflective band in children and adults.

The thickness and number of IRLs at the foveal center decrease over time as the premature infant eye matures. This causes deepening of the foveal pit. Most of this thinning occurs by inner retinal cell migration centrifugally and occurs between 31 and 42 weeks of PMA [16, 17]. This further leads to the decrease in the foveal-to-parafoveal inner retinal layer thickness ratio with increasing age. This centrifugal cell migration of the IRL to form the foveal pit has been confirmed on three-dimensional maps. This is consistent with the progressive increase in the height of a parafoveal annulus of the retina. Most of this IRL migration occurs between 31 and 42 weeks PMA.

4.2. Photoreceptor Layer Development [7, 16, 18]. The photoreceptor development is another aspect that has been better understood with the help of SD OCT, by providing a timeline of the development imaged in vivo. The height of the photoreceptor layer increases progressively from infancy to adulthood. This occurs rapidly after 38-week PMA in all the regions and especially in the cone-dense fovea. Furthermore, even if there is a delay in the inner retinal layer migration, this does not appear to affect or delay the development of the PRL complex.

Photoreceptor subelements are subcellular structures that can be imaged with the current available SD OCT devices. These structures include the external limiting membrane (ELM), the IS/OS (inner segment/outer segment) also known as the ellipsoid zone (EZ), and photoreceptor outer segments to RPE (OS-RPE) or interdigitation zone (IZ). The timelines when these layers "appear" or more correctly "can be imaged" varied considerably and are obviously a function of the resolution of the currently available devices. For example, in a study performed in the United States [16], the ELM was not observed until 42 weeks, whereas in a cohort of babies born with a heavier birth weight, it was imaged between 40 41 weeks [5]. 


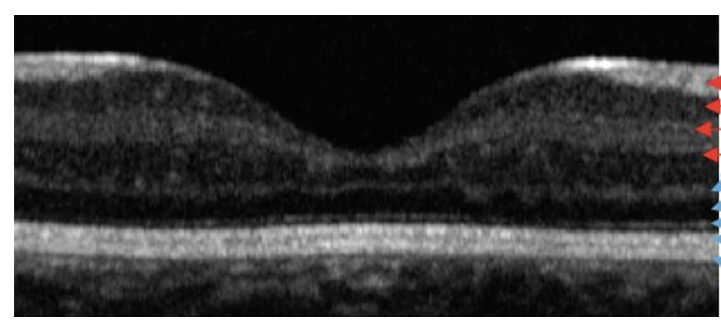

(a)

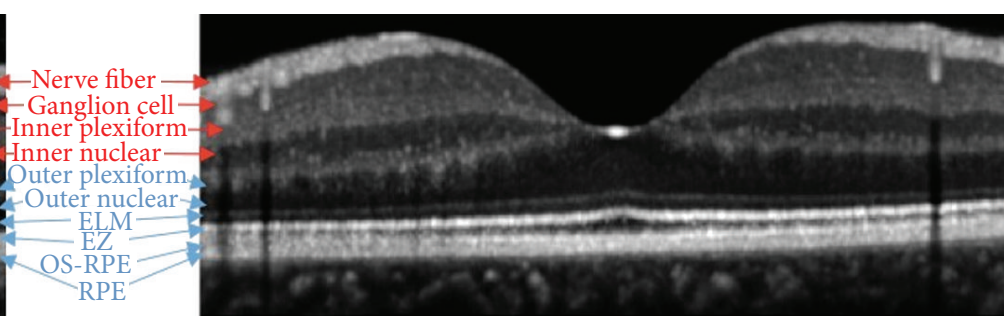

(b)

FIGURE 2: Retinal layers (red are inner retinal layers and blue are outer retinal layers) are compared between PMA 38-week preterm infant (a) and 38-year-old adult (b).

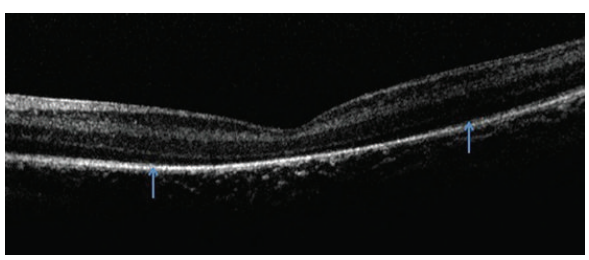

(a) The IS OS band or EZ first appears as a distinct hyperreflective band that first begins above the RPE band and then converges towards the center from the periphery (centripetal growth). At 38 weeks of PMA, two faint shadows (blue arrows) are seen at the two ends of the image

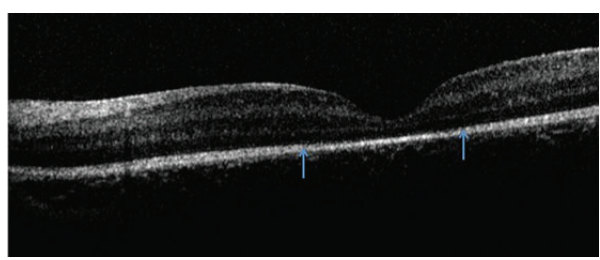

(c) Four more weeks later at 46-week PMA, the two bands are now seen coming closer to each other towards the foveal center

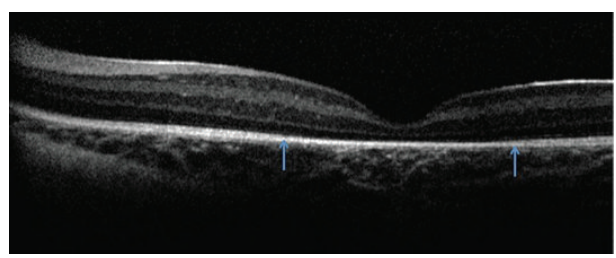

(b) The same infant imaged 4 weeks later at 42 -week PMA, showing a more clearly visible second layer at the periphery (blue arrows)

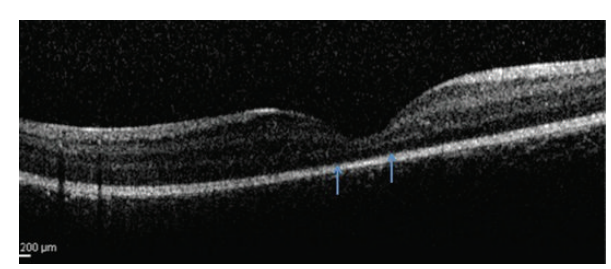

(d) At 47-week PMA the EZ band from either side appears to almost touch at the foveal center

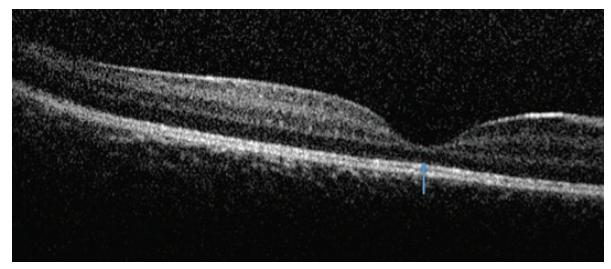

(e) At 48-week PMA in this case, both ends of the EZ band join at the foveal center and appear as a distinct layer

FIgURE 3: The centripetal growth of the IS-OS or EZ band from the periphery to the foveal center at different age groups imaged on the same infant (photo courtesy of Figures 3(a)-3(e) from Figures 90.2-90.6, Page 259-360, Chapter 90 of [59]).

In contrast to adult retinas, the photoreceptor layer in infants is initially thinner in the foveal center. As the infant grows, there is a progressive centripetal growth of the photoreceptor subcellular structures that extend into the foveal center. These layers include the IS-OS or EZ, the ELM, and the OS-RPE, respectively. The EZ band is a low reflective band barely elevated from the RPE outside the fovea imaged as early as 33 weeks PMA, which continuously thickens and moves towards the foveal center. The EZ is at the foveal center in $47 \%$ of term infants but in only $14 \%$ of preterm infants [18]. The process of central growth of the EZ band towards the foveal center at different ages in a premature infant is depicted in Figures 3(a)-3(e).

The RPE layer is seen as a hyperreflective layer after 31 weeks of PMA. The ELM has been variably noted in Asian Indian infants as early as 40.2 weeks and at 42 weeks in Caucasian infants [5]. However the time of imaging may vary with the imaging devices, the protocols, and software 
used in its capture. The OS-RPE or the IZ is the second subtle hyperreflective band between the IS/OS junction and the main RPE reflex. This is believed to be the interface between the photoreceptor outer segments and the RPE microvilli (OS/RPE) and differentiates at the apical side of the RPE much later in childhood. With these changes, the apical microvilli grow and change the interface with the photoreceptor outer segments. The band is not visible over the macula at term birth [18]. It is unclear at this time, about the exact age when this band matures into a layer, and is speculated to happen as late as the end of the first decade or in early adulthood.

A map of retinal layer development in different ages and graphical depiction of foveal development is shown in Figure 4.

4.3. Histological Correlation of SD OCT Findings. Correlation and interpretation of neonatal SD OCT findings have come from histopathology and immunolabeling of postmortem eyes of neonates and children. [19]. Hendrickson et al. showed that at 22 weeks of fetal gestation, the fovea is a five-layered region with a thick GCL and a thin outer nuclear layer. After 25 weeks, the foveal pit begins to develop and invaginates into the INLs. Between fetal weeks 28-37, there is further deepening of the foveal pit, which thins the GCL, IPL, and INL compared to the layers surrounding the pit. Postnatally, the foveal pit is wider and shallower than before birth and has displaced most of the inner layers. The inner segment is longer and narrower than before birth and there is a short outer segment.

Over the postnatal period in the first 1 year of life, the pit becomes wide and shallow with no neurons in the center except for cone cell bodies. The OPL grows in size due to the increased number and length of the cone axons. There is more cone packing in the center as well. The final maturation continues through childhood. The foveal center has thin and long cones and the rod outer segments too become long with eccentricity.

Vajzovic et al. [17] compared the morphology with the layer thickness of the retinal SD OCT validating it as a reliable tool to assess foveal development and disease. The salient features pertinent to the foveal development that were reported are as follow: (1) there is progressive increase in the neurosensory retinal thickness from 30 weeks of postmenstrual age to 16 years and (2) preterms demonstrate shallower foveal pit and short, underdeveloped foveal photoreceptors.

Studies correlating histopathological structure with SD OCT [20-23] help us better understand in vivo anatomy. The foveal pit is the most characteristic feature of the human fovea and is caused by lateral displacement of the IRLs. During this process, the cones are tightly packed and elongate, migrating centripetally to the foveal center. The foveal avascular zone (FAZ) emerges by the 25th week PMA and the pit is also defined around this time. Further during the gestational development, there is inner retinal migration and reduction in the number of ganglion and bipolar cells at the foveal center. In the final trimester of pregnancy, the photoreceptors are more mature in the parafoveal and perifoveal area compared to the foveal center and the RPE interdigitates during this time with the outer segment as well. After birth, the foveal pit continues to get remodeled and is believed to reach maturity by 18 months of age. However, photoreceptors continue to elongate in the fovea postnatally with greater maturity in term born infants than very preterm infants imaged at term equivalent age [18]. The photoreceptors are said to reach the lower range of adults by four years of age.

Dubis et al. [24] showed the mapping of normal foveal development using SD OCT and histologic examination. The photoreceptor and foveal pit maturation showed variability even in age-matched individuals. Even through the 43rd week PMA, the IRLs persisted although the pit deepened and widened. In their series, the youngest infant with a fused inner retina with complete excavation of all inner retinal layers was 52nd week PMA or the third corrected month (assuming 40 weeks as term gestation). The outer retina in their series resembled adult-like bands on the SD OCT at 17 months of age.

Vajzovic et al. recently compared the photoreceptor development on SD OCT of preterm infants born with a gestational age of $<32$ weeks with that of term infants. Preterm infants between the PMAs of 37-42 weeks were imaged. The EZ developed in the foveal center only in $14 \%$ of preterm babies compared to $47 \%$ of the term babies. This was fewer in infants with macular edema than those without. For those with incomplete EZ development, the distance from the foveal center was less in term infants (mean 492 microns) compared to preterm infants (mean 783 microns). The cone outer segment tips (COST) layer was not seen in any infant in the age group studied. The study highlights the fact that photoreceptor inner and outer segment development is delayed in preterm infants compared to term infants and may actually explain the differences in visual function in them [18].

\section{Spectral Domain Optical Coherence Tomography in Disease}

As majority of the reports thus far pertain to the study of SD OCT in retinopathy of prematurity, this will be discussed in detail and then other non-ROP conditions will be summarized.

5.1. SD OCT in Retinopathy of Prematurity. The gold standard for ROP screening and management has been the examination of infants with the indirect ophthalmoscope. However, fundus imaging using wide-field retinal cameras has offered several advantages including providing telemedicine and remote screening of these infants as well as for medicolegal documentation, training, and education [25-28]. More recently, SD OCT imaging has been reported to demonstrate clinically unseen or poorly detected retinal features $[3,6,10]$, which include the following:

(1) Preretinal structures are useful in detecting neovascularization $(\mathrm{NV})$ or posterior $\mathrm{NV}$ which often accompany the more aggressive forms of zone 1 disease or aggressive posterior ROP (APROP) $[3,6]$. 

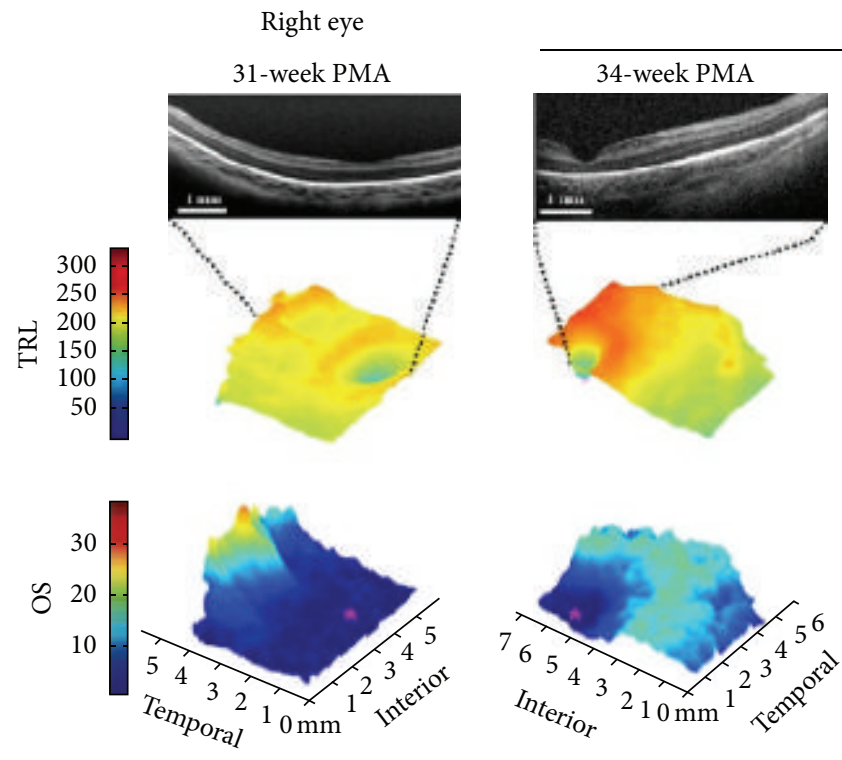

Left eye of the same premature infant
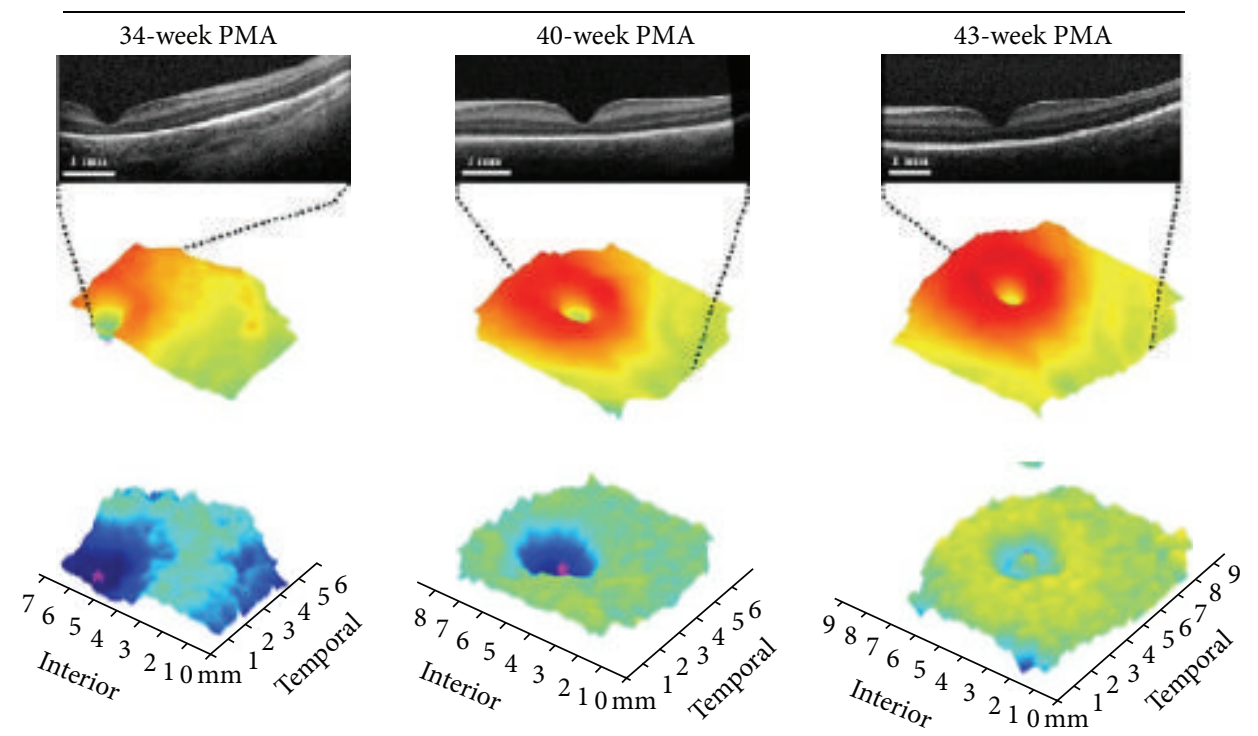

(a)
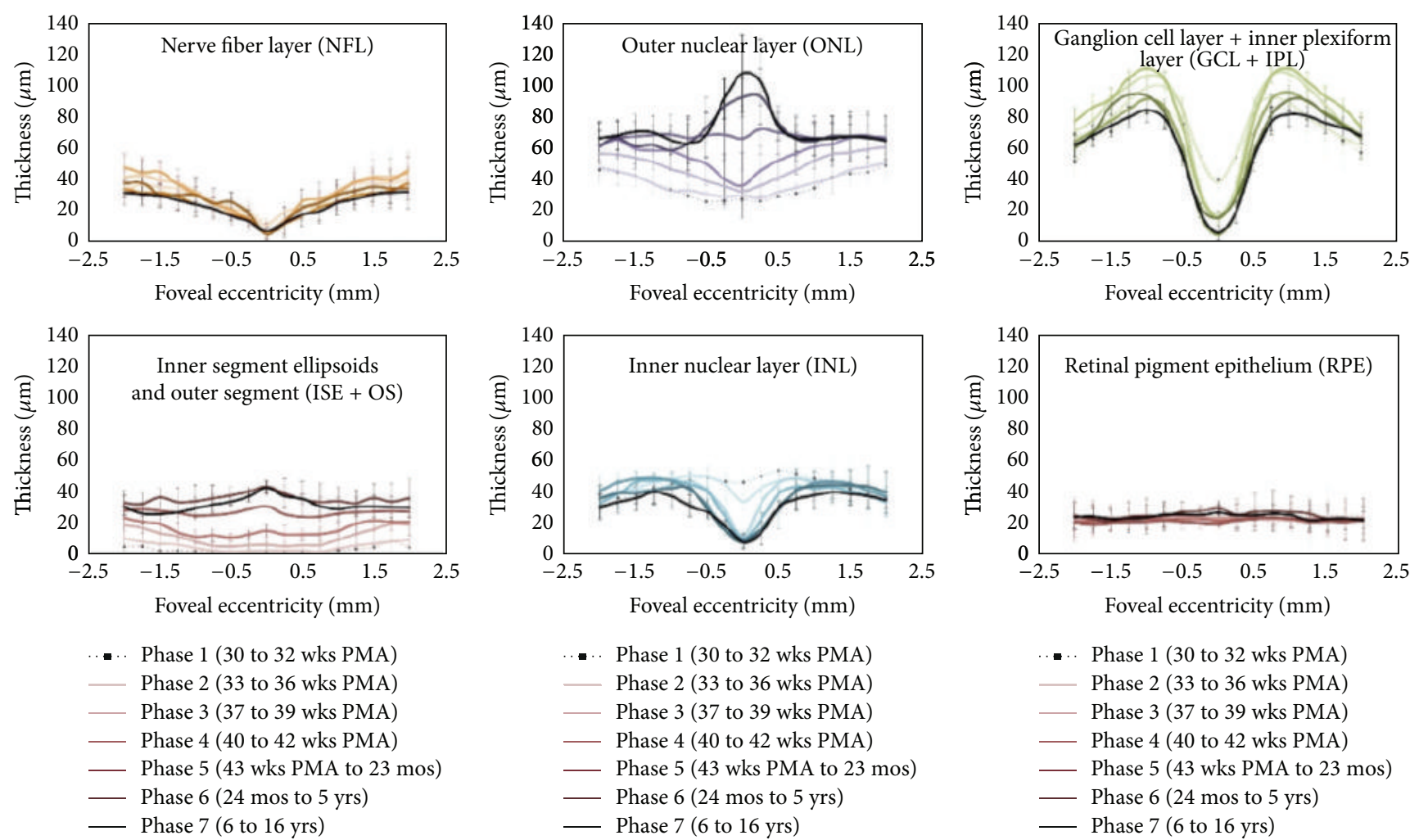

-.. Phase 1 (30 to 32 wks PMA) Phase 2 (33 to 36 wks PMA)

— Phase 3 (37 to 39 wks PMA)

— Phase 4 (40 to 42 wks PMA)

- Phase 5 (43 wks PMA to 23 mos)

— Phase 6 (24 mos to 5 yrs)

— Phase 7 (6 to 16 yrs)

-. Phase 1 (30 to 32 wks PMA) Phase 2 (33 to 36 wks PMA)

— Phase 3 (37 to 39 wks PMA)

— Phase 4 (40 to 42 wks PMA)

— Phase 5 ( 43 wks PMA to 23 mos)

— Phase 6 (24 mos to $5 \mathrm{yrs}$ )

— Phase 7 (6 to 16 yrs)

(b)

FIGURE 4: 3D map of retinal layers and their dynamic changes with age in a neonate. The lower portion of the image has a segment on inner segment ellipsoid (photo courtesy: for the top of the figure (color maps), taken from Figure 2(a), Page 2320 of [16]; for the bottom portion of the figure (graphs), taken from Figure 2, Page 782. of [17]). 


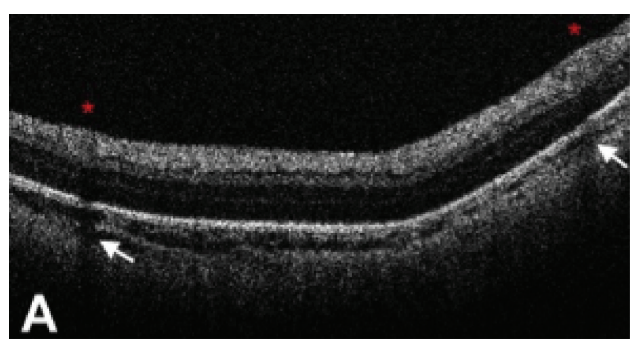

(a)

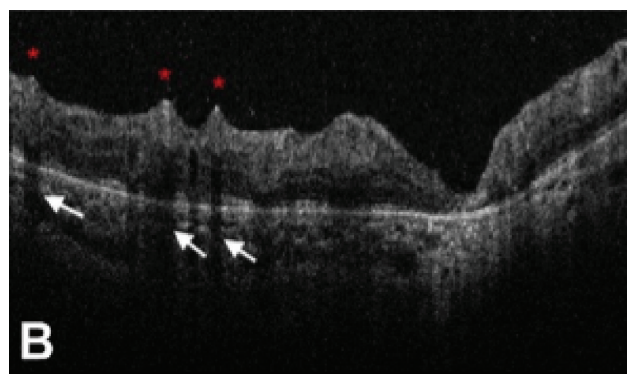

(b)

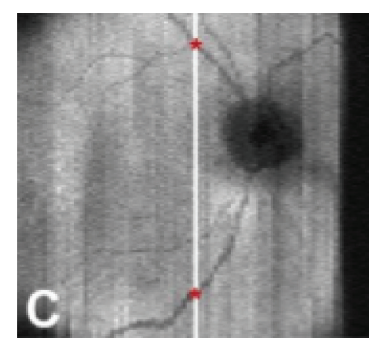

(c)

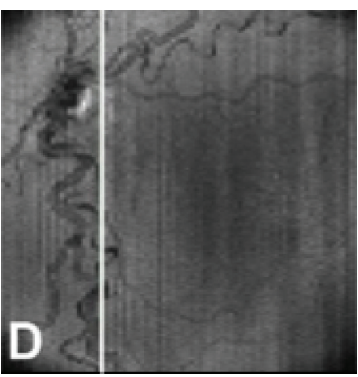

(d)

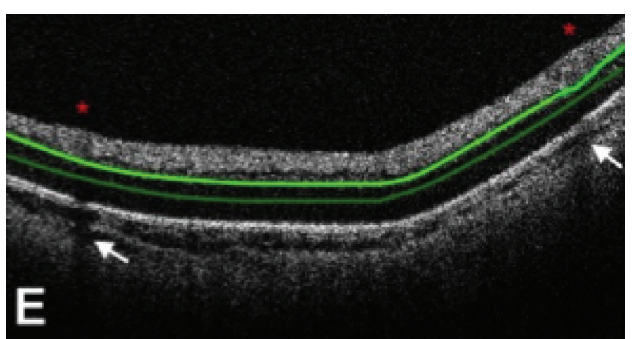

(e)

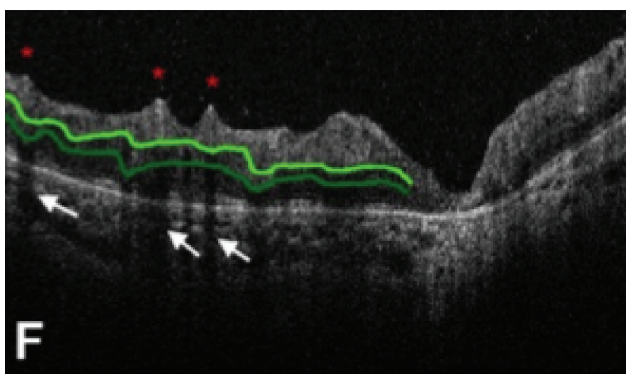

(f)

FIGURE 5: The spectral domain optical coherence tomography (SD-OCT) scans from a 31-week postmenstrual age (PMA) neonate (a) with retinopathy of prematurity (ROP) zone II, stage 2 , and normal vasculature per clinical examination and a 48 -week PMA neonate (b) with ROP zone II, stage 3, and plus disease. No vessel elevation is seen in (a) and severe vessel elevation is seen in (b). Retinal images (c, d) created from axial compression of SD-OCT scans. (e) and (f) contain the same scans as (a) and (b), respectively, but highlight the smooth retinal layer contour in (e) and the scalloped pattern on (f). Red asterisks are placed over vessels, and the corresponding location on the retinal image is shown on (c) and (d). White arrows point to shadow produced by the corresponding vessels. ((e) and (f)) The top light green line represents the inner plexiform layer, and the bottom dark green line represents the outer plexiform layer. (Photo courtesy Figure 2, Page 129, of [32].)

(2) Clinically undetected structures that have been imaged on SD OCT include retinoschisis [29], vitreoretinal interface and epiretinal membranes [30], retinal detachment [6], retinal pigment epithelium changes, and atrophy [30].

(3) OCT has also been used to differentiate and prognosticate macular involvement in advanced cases of ROP, which can potentially change the diagnosis from stage $4 \mathrm{~A}$ to $4 \mathrm{~B}[2]$.

(4) Postsurgery, after lens sparing vitrectomy, SD OCT has been used to monitor the reattachment of the macula, enabling better prognostication and patient education and allowing the correlation between structural and visual improvement [31].

(5) OCT guided laser photoablation of residual neovascular fronds that are difficult to detect on clinical exam after the initial laser in extensive cases of APROP has been reported in Asian Indian infants [4].

(6) In an attempt to correlate SD OCT features of ROP with vascular changes documented on fundus images, Maldonado et al. [32] have investigated the role of 3D-volume analysis and proposed the vascular abnormality score by OCT (VASO). The score is based on the presence of at least one of the following features on SD OCT: (a) retinal vessel elevation, (b) scalloped retinal layers, (c) hyporeflective vessels, and (d) retinal spaces. The score was noted to be higher in eyes with plus disease than those without. These infants had a greater retinal surface elevation, which matched with en face retinal vascular patterns giving us the evidence that vascular dilatation and tortuosity effect perivascular tissue (Figure 5).

5.2. SD OCT and Macular Edema. Macular edema or foveal edema has been the subject of recent research since it was incidentally detected in clinically normal looking foveae. Owing to the current lack of evidence of the exact etiology, extent, course, and effect of this entity, there is considerable scope for more research on this subject.

Vinekar et al. [5,7] detected what they described as "foveal disruptive" changes on the SD OCT in clinically normal looking foveae in a cohort of Asian Indian premature infants undergoing ROP screening. These changes were detected on a modified hand-held version of a standard, adult, tabletop OCT device, Spectralis (Heidelberg, Germany). Of the 79 eyes included in the study with clinical normal foveae and Stage 2 ROP, 23 (29.1\%) eyes appeared to have abnormal foveal changes characterized as "Pattern A" which involved a dome shaped foveal elevation and cystoid spaces with highly reflective intervening vertical septae observed in 12 (52.2\%) eyes. "Pattern B," which was characterized by preservation of the foveal depression with fewer intraretinal cystoid spaces was seen in 11 (47.8\%). "Abnormal foveal changes" were observed in infants with Stage 2 ROP and not in Stage 1 ROP or non-ROP infants. These changes peaked at 37 PMA and 
TABLE 2: Comparison of macular edema in premature infants reported by two groups in the United States of America and India, respectively.

\begin{tabular}{|c|c|c|}
\hline & $\begin{array}{l}\text { Maldonado et al. [16]* } \\
\text { Rothman et al. [9] }\end{array}$ & $\begin{array}{c}\text { Vinekar et al. [7] } \\
\text { Vinekar et al. [31] }{ }^{* * *} \\
\end{array}$ \\
\hline Number of patients & 42 & 74 \\
\hline Birth weight (mean) & 760 grams & 1282 grams \\
\hline Gestational age (mean) & 26 weeks & 31 weeks \\
\hline Race & $\begin{array}{c}\text { Mixed race } \\
\text { (African American 52\%, White 40\%, and } \\
\text { Hispanic 7\%) }\end{array}$ & Asian Indian (100\%) \\
\hline ROP stages included & $0,1,2$ and 3 & 0,1 and 2 \\
\hline Incidence of macular edema, $n(\%)$ & $21(50)$ & $\begin{array}{c}12 \text { (16) } \\
\text { (23 eyes of } 146 \text { eyes with ROP) }\end{array}$ \\
\hline CME characteristics & Bulging fovea 13 (62) & $\begin{array}{l}\text { Pattern A 52\% } \\
\text { Pattern B } 48 \%\end{array}$ \\
\hline Central foveal thickness & 166 (91-499) microns & 206 (108-304) microns \\
\hline CME resolution (latest weeks) & $\begin{array}{c}43^{*} \\
9 \text { months }\end{array}$ & 52 \\
\hline Visual acuity correlation (earliest checked) & 9 months** & 3 months ${ }^{* * *}$ \\
\hline Age range of visual correlation & 9 months -5 years, (retrospective) & 3-12 months (prospective) \\
\hline Visual acuity mean at 9 months in logMAR & $\begin{array}{l}\text { With edema (2): OD = } 1.3,1.3 \\
\text { OS }=1.9,1.3 \\
\text { Without edema }(2): \text { OD }=0.8,1 \\
\text { OS }=1,1\end{array}$ & $\begin{array}{c}\text { Edema }+ \text { ROP }(11)=1.30 \\
\text { Without edema }+ \text { ROP }(16)=1.20 \\
\text { Preterm }(17)=0.98\end{array}$ \\
\hline
\end{tabular}

all self-resolved without therapy or intervention by the 52 nd week PMA or the third corrected month. Two etiologies were proposed, one of increased vascular endothelial growth factor (VEGF) and the other a mechanical factor. Ethnic variability and the clinical relevance are still under investigation.

Similar "macular edema like" changes have been reported in the Caucasian and mixed ethnic cohorts in North America [10-12]. Lee et al. [30] reported cystoid changes in the inner nuclear layer of $39 \%$ of the OCT sessions in their study of 76 eyes. None of these changes were detected clinically on routine indirect ophthalmoscopy. The difference in macular edema in the Asian Indian [7, 31] and the Caucasian population $[9,16]$ is summarized in Table 2 .

Maldonado et al. in 2012 [33] studied the association of severity of cystoid macular edema (CME) with ROP and other systemic health factors unrelated to ROP. They too hypothesized that edema could be a marker of elevated active intravitreal VEGF. Forty-two infants were assessed for the severity of CME. The measures of severity included CFT, retinal layer thickness and foveal-to-parafoveal ratio. These parameters were correlated with the stage of ROP, plus disease, and treatment status as well as systemic factors such as Apgar score, surgery for patent ductus arteriosus, culture-proven sepsis, surgery for necrotizing enterocolitis, and the presence of intraventricular hemorrhage, periventricular leukomalacia, bronchopulmonary dysplasia, or hydrocephalus. They found that $50 \%$ of these infants had edema irrespective of the severity of ROP or the systemic factors under consideration.

Extending the finding of these edemas in more advanced stages of ROP, Dubis et al. [34] in a prospective, observational case series evaluated subclinical macular findings in premature infants at risk of ROP using the hand held system. This study demonstrated the presence of CME in 25 of the 49 infants (51\%) imaged in the neonatal intensive care unit. The edema was found in Stages 0, 1, 2, 3, and 4A of ROP thereby concluding that the stage of the disease may not be associated with macular edema thereby concurring with the findings of Maldonado et al. [33].

More recently, Rothman et al. [35] evaluated the association of CME and neurodevelopmental outcomes in very preterm infants at 18 to 24 months corrected age. The imaging was done during routine ROP screening and the Bayley scores for neuro- and cognitive development were assessed at 18 to 24 months. Of the 53 children evaluated with Bayley scores, 31 children who had CME as infants had a lower mean score on cognitive, motor, and language subscale when compared to the children who did not have edema as infants. This paper throws light on the association of cystoid macular edema and neurodevelopment and the possible potential for retinal examination of macular edema as an indicator of neurodevelopmental health in infants.

Erol et al. [36] in a study from Turkey reported macular edema in 139 eyes of 190 premature infants imaged. Of their study cohort 126 eyes had ROP. They noted that $54 \%$ of eyes with ROP had edema compared to $31 \%$ without ROP. With increasing stage of ROP, there was a higher incidence of macular edema, that is, $46.3 \%$ in stage $1,57.1 \%$ in stage 2 , and $87.5 \%$ in stage 3 ROP.

Recently, Vinekar et al. [31] reported the visual and refractive outcome of infants with macular edema by following their initial cohort of infants with edema and comparing them with age matched premature infants with ROP (positive control) and without ROP (negative controls). They found 
that the visual acuity was lower in infants with macular edema compared to the other two control groups throughout the study period of one year, but statistically significant only at 3 months. The edema cohort was more hyperopic compared to the other two groups between 3 and 12 months of age and was possibly due to visual disturbances caused at a critical time of foveal development. On the other hand, in a small series including infants with and without edema, Rothman et al. [9] correlated the posterior segment microanatomy from perinatal SD OCT to visual acuity, brain abnormalities, and neurodevelopment. Those without edema had better vision compared to those with edema. They also found sensorimotor deficits and neurodevelopment changes in the group with edema. In one infant in the study the edema persisted through nine months.

5.3. Shaken Baby Syndrome (SBS) [10, 37-39]. SD OCT provides valuable information in SBS. Vitreoretinal membranes seen on imaging support the direct mechanical effect as one of the main pathophysiological theories. Preretinal blood, localized vitreous detachment, premacular folds, and attachment of the vitreous to the ILM at the apices of the perimacular folds are features that are detected. The presence of perimacular folds and hemorrhagic macular retinoschisis has been linked to poor visual outcome [10].

5.4. SD OCT in Other Retinal Conditions in Infancy. Other retinal conditions in infants and young children that have been reported are combined hamartoma of the retina and RPE [40-45], incontinentia pigmenti, retinal dystrophies and degenerations [46-55], retinoschisis, and other syndromes [56]. Systemic conditions like liver failure and neonatal hemochromatosis [57], causing macular changes, suggest that OCT can image retinal manifestations of ocular and systemic disease even when it is not clinically discernible [58].

\section{SD OCT and the Choroid}

SD OCT studies of the choroid have been extensive in adults, especially with the use of techniques such as enhanced depth imaging (EDI) that provides better visualization of the layers. However, there have been only a handful of studies published, which study the choroid in infants and children [60, 61]. Park and Oh analyzed the choroidal thickness profiles of children between the ages of 4 and 10 years subdividing them into preterm and full-term children [60]. From the 31 preterm and 30 full-term born children it was found that those that belonged to the preterm group had decreased choroidal thickness $3 \mathrm{~mm}$ temporal to the fovea.

More recently, Moreno et al. reported the feasibility of choroidal imaging without the use of EDI in both preterm and term infants and concluded that the presence of melanin in the RPE and less developed pigmentation in the choroid at that early age does not hinder choroidal imaging in preterm infants without an advanced stage of ROP. It was also noted that the choroidal thickness increased with age. However preterm infants had a thinner choroid when compared to term infants at the same age and adults [61].

\section{SD OCT and the Optic Nerve}

Our knowledge and understanding of the optic nerve and its development so far come from various histological studies [62]. With the advent of the portable hand-held SD OCT we can now perform imaging at the clinic, providing us with realtime information of the optic nerve. This technology has led to a number of studies of the optic nerve in adults; however the application of SD OCT to study the infant optic nerve is still limited.

OCT studies have looked at the development of the optic nerve and its association with possible demographic factors. The study by Allingham et al. [63] looked at the variation in the optic nerve parameters in full-term infants of different races and found a significant difference in the cup-disc ratio at the time of birth between white, black, and Hispanic babies which could be a normal trend for each of the races, thereby defining a normative range among the newborns.

Another study by Tong et al. [64] described the optic nerve parameters in term and preterm infants and their association with neurodevelopment and prematurity. The study showed that preterm infants with a risk of developing ROP had a larger cup-disc ratio when compared to term infants. In another study, the average retinal nerve fiber layer (RNFL) thicknesses in healthy, full-term neonates has been assessed and found to be normally distributed at $1.5 \mathrm{~mm}$ radial distance from the optic nerve. Interestingly, they found no significant difference of RNFL thickness with respect to race, gender, gestational age, or birth weight [65].

\section{SD OCT and Visual Acuity Correlation in Infants}

Although infant SD OCT imaging helps us better understand the anatomy, subclinical pathology and foveal development in normals and in disease, there is lack of evidence that correlates visual acuity with these retinal structures demonstrated on OCT. A retrospective analysis of 62 (54.4\%) premature infants who had their visual acuities correlated with foveal layers at different time intervals in the first year of life showed that visual acuity correlated positively with IRL fusion, presence of the ELM, and the outer segment layer [66].

In another prospective study, 50 Asian Indian premature infants with and without ROP were followed through their first year of life and imaged at regular intervals of 3 months. The group without ROP showed better visual acuity correlation with foveal layer development compared to those without foveal layer maturation. In the group with ROP, there was no significant difference between those with and without these layers [67]. Future multicenter and multiethnic studies that evaluate the influence of foveal development and visual acuity with and without disease are required.

\section{Future Trends}

With the expanding knowledge base on spectral domain optical coherence tomography in the practice of pediatric retinal disease, future trends are likely to include custom software to create three-dimensional data sets to map structures, shapes, 
and abnormalities which would provide spatial information for understanding normal anatomy, development, and pathologic events [68].

Newer imaging tools such as the OCT-angiography, adaptive optics, and oximetry are likely to be modified for the use in pediatric retina, much like the evolution of the hand-held OCT now in vogue for infants in the clinic setting. Intraoperative use of microscope integrated OCT devices [69] is likely to provide a surgical assist for better demonstration of the pathology during and in the immediate postoperative period. OCT is a step beyond the routine fundus imaging and has allowed us to see the unseen and learn the unknown. Further evolution of the technology is most certainly going to help us understand and manage our patients better.

\section{Abbreviations}

APROP: Aggressive posterior retinopathy of prematurity

CCD: Charge coupled device

CFT: Central foveal thickness

CME: Cystoid macular edema

COST: Cone outer segment tips

EDI: $\quad$ Enhanced depth imaging

ELM: External limiting membrane

EZ: $\quad$ Ellipsoid zone

FAZ: Foveal avascular zone

GCL: Ganglion cell layer

ILM: Inner limiting membrane

INL: Inner nuclear layer

IPL: Inner plexiform layer

IRL: Inner retinal layers

IS-OS: Inner segment/outer segment

IZ: Interdigitation zone

NV: Neovascularization

OPL: $\quad$ Outer plexiform layer

ORL: Outer retinal layers

OS-RPE: Photoreceptor outer segments to RPE

PMA: Postmenstrual age

PRL: $\quad$ Photoreceptor layer

RNFL: Retinal nerve fiber layer

ROP: Retinopathy of prematurity

RPE: Retinal pigment epithelium

SD OCT: Spectral domain optical coherence tomography

VEGF: Vascular endothelial growth factor.

\section{Conflict of Interests}

Cynthia A. Toth received research support for this work through her university from The National Institutes of Health, The Hartwell Foundation, Research to Prevent Blindness, the Andrew Family Charitable Foundation, and The Retina Research Foundation. Dr. Toth received grants from National Institutes of Health and from Genentech and a loan of equipment from Bioptigen for research unrelated to this study. Dr. Toth receives royalties for surgical technology licensed to Alcon Laboratories. The rest of the authors have no financial interests to declare.

\section{Acknowledgments}

The authors thank Sivakumar Munusamy, Krishnan Narasimha, and Du Tran-Viet for help in imaging and image analysis.

\section{References}

[1] A. Vinekar, "Spectral domain OCT in infants," in Pediatric Retina, M. E. Hartnett, Ed., pp. 112-117, Wolters Kluwer, 2013.

[2] C. K. Patel, "Optical coherence tomography in the management of acute retinopathy of prematurity," American Journal of Ophthalmology, vol. 141, no. 3, pp. 582-584, 2006.

[3] M. M. Joshi, M. T. Trese, and A. Capone Jr., "Optical coherence tomography findings in stage $4 \mathrm{~A}$ retinopathy of prematurity: a theory for visual variability," Ophthalmology, vol. 113, no. 4, pp. 657-660, 2006.

[4] A. Vinekar, M. Sivakumar, R. Shetty et al., "A novel technique using spectral-domain optical coherence tomography (Spectralis, SD-OCT+HRA) to image supine non-anaesthetized infants: Utility demonstrated in aggressive posterior retinopathy of prematurity," Eye, vol. 24, no. 2, pp. 379-382, 2010.

[5] A. Vinekar, K. Avadhani, M. Sivakumar et al., "Macular edema in premature infants," Ophthalmology, vol. 119, no. 6, pp. 12881290.e1281, 2012.

[6] S. H. Chavala, S. Farsiu, R. Maldonado, D. K. Wallace, S. F. Freedman, and C. A. Toth, "Insights into advanced retinopathy of prematurity using handheld spectral domain optical coherence tomography imaging," Ophthalmology, vol. 116, no. 12, pp. 2448-2456, 2009.

[7] A. Vinekar, K. Avadhani, M. Sivakumar et al., "Understanding clinically undetected macular changes in early retinopathy of prematurity on spectral domain optical coherence tomography," Investigative Ophthalmology and Visual Science, vol. 52, no. 8, pp. 5183-5188, 2011.

[8] R. S. Maldonado, J. A. Izatt, N. Sarin et al., "Optimizing handheld spectral domain optical coherence tomography imaging for neonates, infants, and children," Investigative Ophthalmology and Visual Science, vol. 51, no. 5, pp. 2678-2685, 2010.

[9] A. L. Rothman, D. Tran-Viet, L. Vajzovic et al., "Functional outcomes of young infants with and without macular edema," Retina, 2015.

[10] A. W. Scott, S. Farsiu, L. B. Enyedi, D. K. Wallace, and C. A. Toth, "Imaging the infant retina with a hand-held spectral-domain optical coherence tomography device," The American Journal of Ophthalmology, vol. 147, no. 2, pp. 364.e2-373.e2, 2009.

[11] G. T. Chong, S. Farsiu, S. F. Freedman et al., "Abnormal foveal morphology in ocular albinism imaged with spectral-domain optical coherence tomography," Archives of Ophthalmology, vol. 127, no. 1, pp. 37-44, 2009.

[12] A. Cook, S. White, M. Batterbury, and D. Clark, "Ocular growth and refractive error development in premature infants with or without retinopathy of prematurity," Investigative Ophthalmology and Visual Science, vol. 49, no. 12, pp. 5199-5207, 2008.

[13] R. A. Gordon and P. B. Donzis, "Refractive development of the human eye," Archives of Ophthalmology, vol. 103, no. 6, pp. 785789,1985

[14] S. J. Isenberg, M. Del Signore, A. Chen, J. Wei, and P. D. Christenson, "Corneal topography of neonates and infants," Archives of Ophthalmology, vol. 122, no. 12, pp. 1767-1771, 2004. 
[15] E. M. Anger, A. Unterhuber, B. Hermann et al., "Ultrahigh resolution optical coherence tomography of the monkey fovea. Identification of retinal sublayers by correlation with semithin histology sections," Experimental Eye Research, vol. 78, no. 6, pp. 1117-1125, 2004.

[16] R. S. Maldonado, R. V. O’Connell, N. Sarin et al., "Dynamics of human foveal development after premature birth," Ophthalmology, vol. 118, no. 12, pp. 2315-2325, 2011.

[17] L. Vajzovic, A. E. Hendrickson, R. V. O'Connell et al., "Maturation of the human fovea: correlation of spectral-domain optical coherence tomography findings with histology," American Journal of Ophthalmology, vol. 154, no. 5, pp. 779-789, 2012.

[18] L. Vajzovic, A. L. Rothman, D. Tran-Viet, M. T. Cabrera, S. F. Freedman, and C. A. Toth, "Delay in retinal photoreceptor development in very preterm compared to term infants," Investigative Ophthalmology \& Visual Science, vol. 56, no. 2, pp. 908913, 2015.

[19] A. Hendrickson, D. Possin, L. Vajzovic, and C. A. Toth, "Histologic development of the human fovea from midgestation to maturity," American Journal of Ophthalmology, vol. 154, no. 5, pp. 767-778.e762, 2012.

[20] C. Yuodelis and A. Hendrickson, "A qualitative and quantitative analysis of the human fovea during development," Vision Research, vol. 26, no. 6, pp. 847-855, 1986.

[21] A. E. Hendrickson and C. Yuodelis, "The morphological development of the human fovea," Ophthalmology, vol. 91, no. 6, pp. 603-612, 1984.

[22] C. Diaz-Araya and J. M. Provis, "Evidence of photoreceptor migration during early foveal development: a quantitative analysis of human fetal retinae," Visual neuroscience, vol. 8, no. 6, pp. 505-514, 1992.

[23] I. Abramov, J. Gordon, A. Hendrickson, L. Hainline, V. Dobson, and E. LaBossiere, "The retina of the newborn human infant," Science, vol. 217, no. 4556, pp. 265-267, 1982.

[24] A. M. Dubis, D. M. Costakos, C. D. Subramaniam et al., "Evaluation of normal human foveal development using optical coherence tomography and histologic examination," Archives of Ophthalmology, vol. 130, no. 10, pp. 1291-1300, 2012.

[25] A. Vinekar, C. Gilbert, M. Dogra et al., "The KIDROP model of combining strategies for providing retinopathy of prematurity screening in underserved areas in India using wide-field imaging, tele-medicine, non-physician graders and smart phone reporting," Indian Journal of Ophthalmology, vol. 62, no. 1, pp. 41-49, 2014.

[26] G. E. Quinn, G. S. Ying, E. Daniel et al., "Validity of a telemedicine system for the evaluation of acute-phase retinopathy of prematurity," JAMA Ophthalmology, vol. 132, no. 10, pp. 1178-1184, 2014.

[27] A. Vinekar, I. Govindaraj, C. Jayadev et al., "Universal ocular screening of 1021 term infants using wide-field digital imaging in a single public hospital in India - a pilot study," Acta Ophthalmologica, 2015.

[28] A. Vinekar, C. Jayadev, and N. Bauer, "Need for telemedicine in retinopathy ofprematurity in middle-income countries," JAMA Ophthalmology, vol. 133, no. 3, pp. 360-361, 2015.

[29] R. H. Muni, R. P. Kohly, A. C. Charonis, and T. C. Lee, "Retinoschisis detected with handheld spectral-domain optical coherence tomography in neonates with advanced retinopathy of prematurity," Archives of Ophthalmology, vol. 128, no. 1, pp. 57-62, 2010.

[30] A. C. Lee, R. S. Maldonado, N. Sarin et al., "Macular features from spectral-domain optical coherence tomography as an adjunct to indirect ophthalmoscopy in retinopathy of prematurity," Retina, vol. 31, no. 8, pp. 1470-1482, 2011.

[31] A. Vinekar, S. Mangalesh, C. Jayadev et al., "Influence of macular edema on vision in premature infants: 1 year study," Indian Journal of Ophthalmology. In press.

[32] R. S. Maldonado, E. Yuan, D. Tran-Viet et al., "Three-dimensional assessment of vascular and perivascular characteristics in subjects with retinopathy of prematurity," Ophthalmology, vol. 121, no. 6, pp. 1289-1296, 2014.

[33] R. S. Maldonado, R. O’Connell, S. B. Ascher et al., "Spectraldomain optical coherence tomographic assessment of severity of cystoid macular edema in retinopathy of prematurity," Archives of Ophthalmology, vol. 130, no. 5, pp. 569-578, 2012.

[34] A. M. Dubis, C. D. Subramaniam, P. Godara, J. Carroll, and D. M. Costakos, "Subclinical macular findings in infants screened for retinopathy of prematurity with spectral-domain optical coherence tomography," Ophthalmology, vol. 120, no. 8, pp. 1665-1671, 2013.

[35] A. L. Rothman, D. Tran-Viet, K. E. Gustafson et al., "Poorer neurodevelopmental outcomes associated with cystoid macular edema identified in preterm infants in the intensive care nursery," Ophthalmology, vol. 122, no. 3, pp. 610-619, 2015.

[36] M. K. Erol, O. Ozdemir, D. T. Coban et al., "Macular findings obtained by spectral domain optical coherence tomography in retinopathy of prematurity," Journal of Ophthalmology, vol. 2014, Article ID 468653, 7 pages, 2014.

[37] V. Sturm, K. Landau, and M. N. Menke, "Optical coherence tomography findings in Shaken Baby syndrome," American Journal of Ophthalmology, vol. 146, no. 3, pp. 363-368, 2008.

[38] R. H. Muni, R. P. Kohly, E. H. Sohn, and T. C. Lee, "Handheld spectral domain optical coherence tomography finding in shaken-baby syndrome," Retina, vol. 30 , no. 4 , supplemet, pp. S45-S50, 2010.

[39] D. D. Koozekanani, D. V. Weinberg, A. M. Dubis, J. Beringer, and J. Carroll, "Hemorrhagic retinoschisis in shaken baby syndrome imaged with spectral domain optical coherence tomography," Ophthalmic Surgery, Lasers, and Imaging, 2010.

[40] H. M. Kang, H. J. Koh, and E. J. Chung, "Spectral-domain optical coherence tomography of combined hamartoma of the retina and retinal pigment epithelium in neurofibromatosis," Korean Journal of Ophthalmology, vol. 27, no. 1, pp. 68-71, 2013.

[41] N. Goel, B. Pangtey, G. Bhushan, U. K. Raina, and B. Ghosh, "Spectral-domain optical coherence tomography of astrocytic hamartomas in tuberous sclerosis," International Ophthalmology, vol. 32, no. 5, pp. 491-493, 2012.

[42] L. Xu, T. R. Burke, J. P. Greenberg, V. B. Mahajan, and S. H. Tsang, "Infrared imaging and optical coherence tomography reveal early-stage astrocytic hamartomas not detectable by fundoscopy," The American Journal of Ophthalmology, vol. 153, no. 5, pp. 883.e2-889.e2, 2012.

[43] C. S. Huot, K. B. Desai, and V. A. Shah, "Spectral domain optical coherence tomography of combined hamartoma of the retina and retinal pigment epithelium," Ophthalmic Surgery Lasers and Imaging, vol. 40, no. 3, pp. 322-324, 2009.

[44] A. Takahashi, S. Ooto, and N. Yoshimura, "High-penetration optical coherence tomography and enhanced depth imaging in presumed retinal pigment epithelial hamartoma," Retinal Cases and Brief Reports, vol. 7, no. 3, pp. 179-182, 2013. 
[45] A. Vinekar, P. Quiram, N. Sund, J. Wilson, and A. Capone Jr., "Plasmin-assisted vitrectomy for bilateral combined hamartoma of the retina and retinal pigment epithelium: histopathology, immunohistochemistry, and optical coherence tomography," Retinal Cases \& Brief Reports, vol. 3, no. 2, pp. 186-189, 2009.

[46] Y. Makiyama, A. Oishi, A. Otani et al., "Prevalence and spatial distribution of cystoid spaces in retinitis pigmentosa: investigation with spectral domain optical coherence tomography," Retina, vol. 34, no. 5, pp. 981-988, 2014.

[47] T. Duncker, J. P. Greenberg, R. Ramachandran et al., "Quantitative fundus autofluorescence and optical coherence tomography in best vitelliform macular dystrophy," Investigative Ophthalmology and Visual Science, vol. 55, no. 3, pp. 1471-1482, 2014.

[48] H. Lee, V. Sheth, M. Bibi et al., "Potential of handheld optical coherence tomography to determine cause of infantile nystagmus in children by using foveal morphology," Ophthalmology, vol. 120, no. 12, pp. 2714-2724, 2013.

[49] H. M. Kersten, R. H. Roxburgh, N. Child, P. J. Polkinghorne, C. Frampton, and H. V. Danesh-Meyer, "Epiretinal membrane: a treatable cause of visual disability in myotonic dystrophy type 1," Journal of Neurology, vol. 261, no. 1, pp. 37-44, 2014.

[50] J. Zerbib, G. Querques, N. Massamba et al., "Reticular pattern dystrophy of the retina: a spectral-domain optical coherence tomography analysis," American Journal of Ophthalmology, vol. 156, no. 6, pp. 1228-1237, 2013.

[51] S. Salvatore, M. A. Genead, and G. A. Fishman, "The prevalence of macular cysts in patients with clinical cone-rod dystrophy determined by spectral-domain optical coherence tomography," Ophthalmic Genetics, vol. 35, no. 1, pp. 47-50, 2014.

[52] J. Baek, H.-K. Lee, and U. S. Kim, "Spectral domain optical coherence tomography findings in bilateral peripheral cone dystrophy," Documenta Ophthalmologica, vol. 126, no. 3, pp. 247-251, 2013.

[53] S. R. Hannan, G. de Salvo, A. Stinghe, F. Shawkat, and A. J. Lotery, "Common spectral domain OCT and electrophysiological findings in different pattern dystrophies," British Journal of Ophthalmology, vol. 97, no. 5, pp. 605-610, 2013.

[54] A. Vinekar, "Leber's Congenital Amaurosis," in in Spectral Domain Optical Coherence Imaging of the Eye, A. Vinekar and and K. Avadhani, Eds., pp. 372-373, Elsevier, New Delhi, 2013.

[55] A. Vinekar, "Albinism," in Spectral Domain Optical Coherence Imaging of the Eye, A. Vinekar and K. Avadhani, Eds., pp. 361363, Elsevier, New Delhi, India, 2013.

[56] J. Matalia and A. Vinekar, "Rubella retinopathy," in Spectral Domain Optical Coherence Imaging of the Eye, A. Vinekar and K. Avadhani, Eds., pp. 384-385, Elsevier, New Delhi, India, 2013.

[57] R. S. Maldonado, S. F. Freedman, C. M. Cotten, J. M. Ferranti, and C. A. Toth, "Reversible retinal edema in an infant with neonatal hemochromatosis and liver failure," Journal of AAPOS, vol. 15, no. 1, pp. 91-93, 2011.

[58] A. Vinekar, N. Sund, P. Quiram, and A. Capone Jr., "Choroidal neovascular membrane in persistent fetal vasculature syndrome managed with intravitreal pegaptanib sodium in an infant," Retina, vol. 30, no. 4, pp. S41-S44, 2010.

[59] A. Vinekar, R. Maldonado, and C. Toth, "Foveal maturation in infants," in Spectral Domain Optical Coherence Imaging of the Eye, A. Vinekar and K. Avadhani, Eds., pp. 357-360, Elsevier, New Delhi, India, 2013.
[60] K.-A. Park and S. Y. Oh, "Analysis of spectral-domain optical coherence tomography in preterm children: retinal layer thickness and choroidal thickness profiles," Investigative Ophthalmology and Visual Science, vol. 53, no. 11, pp. 7201-7207, 2012.

[61] T. A. Moreno, R. V. O’Connell, S. J. Chiu et al., "Choroid development and feasibility of choroidal imaging in the preterm and term infants utilizing SD-OCT,' Investigative Ophthalmology and Visual Science, vol. 54, no. 6, pp. 4140-4147, 2013.

[62] J. M. Provis, D. van Driel, F. A. Billson, and P. Russell, "Human fetal optic nerve: overproduction and elimination of retinal axons during development," Journal of Comparative Neurology, vol. 238, no. 1, pp. 92-100, 1985.

[63] M. J. Allingham, M. T. Cabrera, R. V. O’Connell et al., "Racial variation in optic nerve head parameters quantified in healthy newborns by handheld spectral domain optical coherence tomography," Journal of AAPOS, vol. 17, no. 5, pp. 501-506, 2013.

[64] A. Y. Tong, M. El-Dairi, R. S. Maldonado et al., "Evaluation of optic nerve development in preterm and term infants using handheld spectral-domain optical coherence tomography," Ophthalmology, vol. 121, no. 9, pp. 1818-1826, 2014.

[65] A. L. Rothman, M. B. Sevilla, S. F. Freedman et al., "Assessment of retinal nerve fiber layer thickness in healthy, full-term neonates," The American Journal of Ophthalmology, vol. 159, no. 4, pp. 803.e2-811.e2, 2015.

[66] A. Vinekar, K. Avadhani, S. Munusamy et al., "Influence of Foveal photoreceptor sub-elements on visual acuity in premature infants with and without retinopathy of prematurity," in Proceedings of the ARVO Annual Meeting, Fort Lauderdale, Fla, USA, May 2012.

[67] A. Vinekar, A. Zope, S. Mangalesh et al., "Correlating foveal spectral domain optical coherence tomography with visual acuity in premature infants with and without retinopathy of prematurity," in Proceedings of the Annual Meeting of the Association for Research in Vision and Ophthalmology (ARVO '15), Denver, Colo, USA, 2015.

[68] R. S. Maldonado and C. A. Toth, "Optical coherence tomography in retinopathy of prematurity: looking beyond the vessels," Clinics in Perinatology, vol. 40, no. 2, pp. 271-296, 2013.

[69] C. Jayadev, S. Dabir, A. Vinekar, U. Shah, T. Vaid, and N. K. Yadav, "Microscope-integrated optical coherence tomography-a new surgical tool in vitreoretinal surgery," Indian Journal of Ophthalmology. In press. 


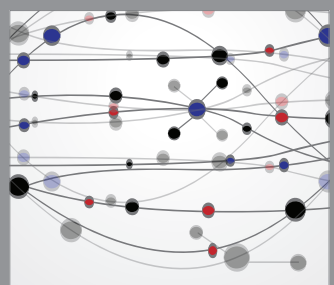

The Scientific World Journal
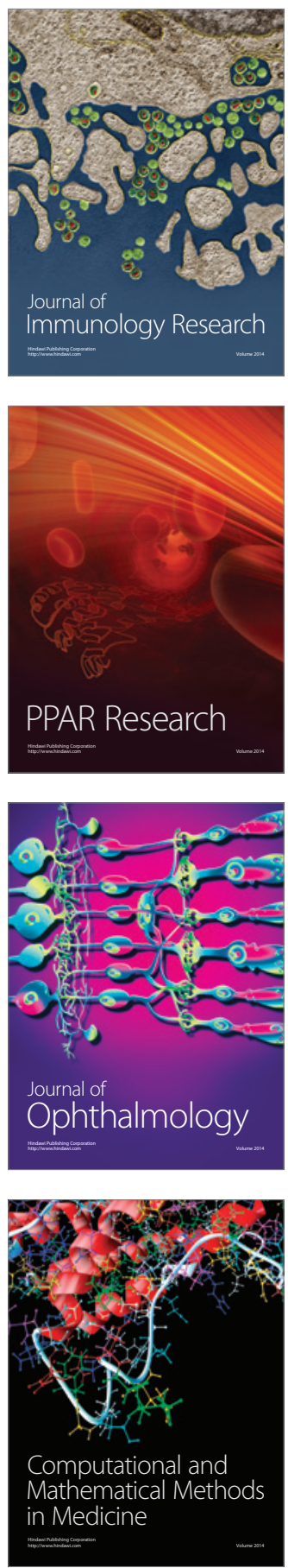

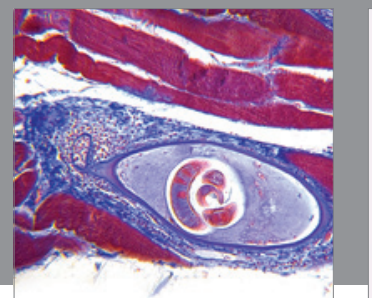

Gastroenterology

Research and Practice
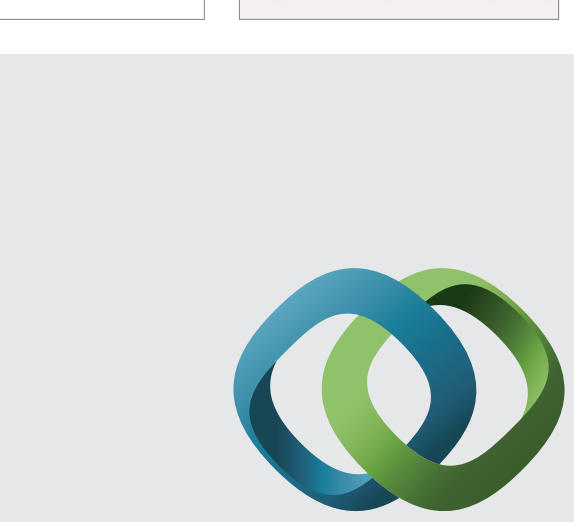

\section{Hindawi}

Submit your manuscripts at

http://www.hindawi.com
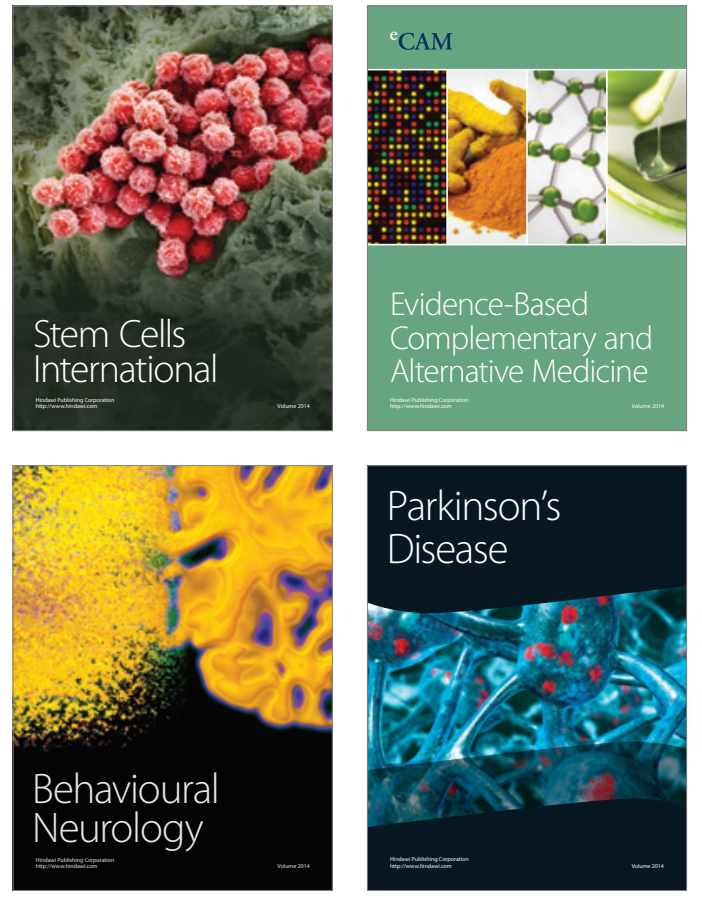
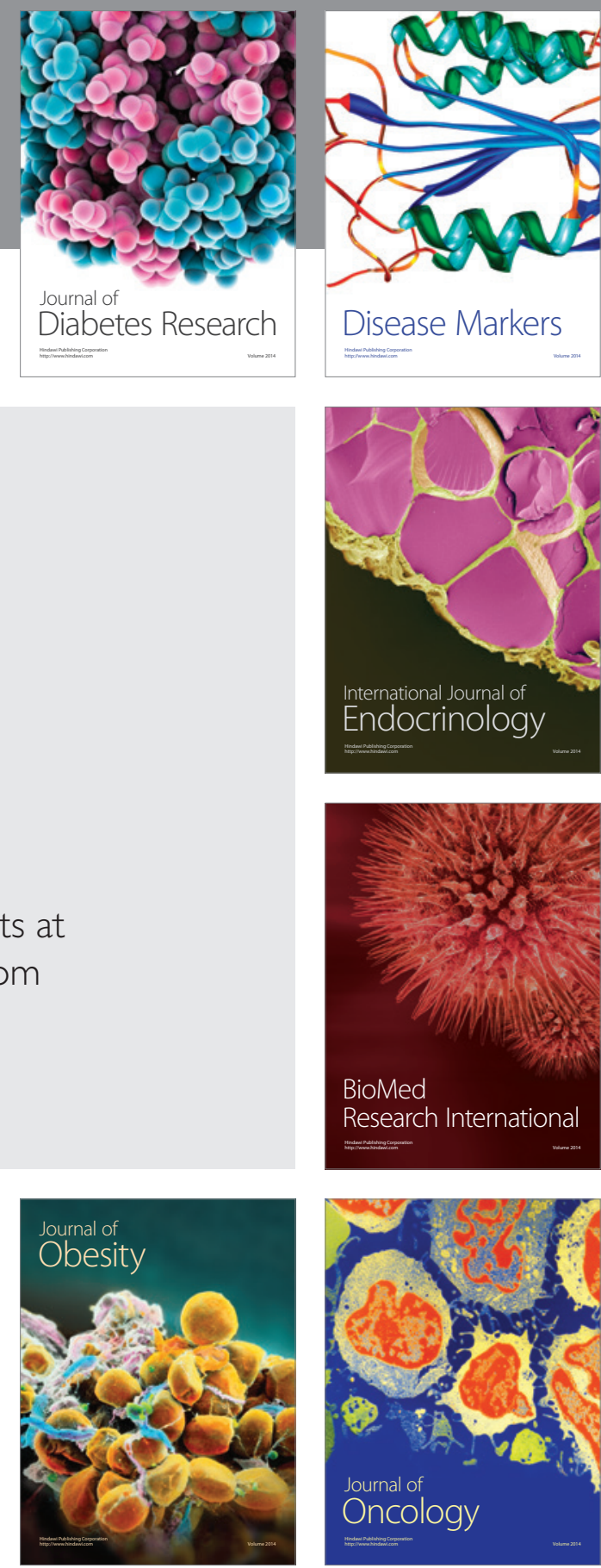

Disease Markers
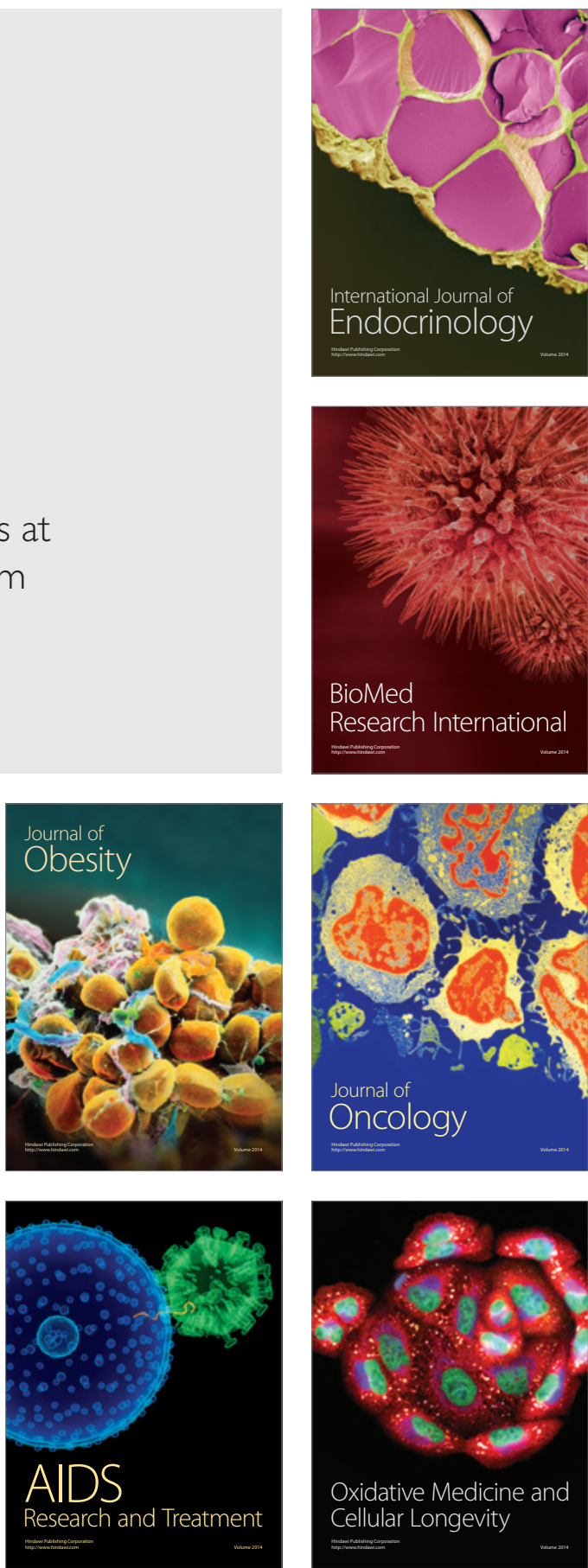